\title{
Observation of diffusive and dispersive profiles of the nonequilibrium polariton-condensate dispersion relation in a $\mathrm{CuBr}$ microcavity
}

\section{Masaaki Nakayama, Masafumi Ueda}

\begin{tabular}{|c|l|}
\hline Citation & Physical Review B. 95(12); 125315 \\
\hline Issue Date & $2017-03-22$ \\
\hline Type & Journal Article \\
\hline Textversion & Publisher \\
\hline & $\begin{array}{l}\text { O2017 American Physical Society. This article may be downloaded for personal use } \\
\text { only. Any other use requires prior permission of the author and American Physical } \\
\text { Society. } \\
\text { The following article appeared in Physical Review B Vol.95, Iss.12 and may be found } \\
\text { at https://doi.org/10.1103/PhysRevB.95.125315 . }\end{array}$ \\
\hline DOI & 10.1103/PhysRevB.95.125315 \\
\hline
\end{tabular}

Self-Archiving by Author(s)

Placed on: Osaka City University 


\title{
Observation of diffusive and dispersive profiles of the nonequilibrium polariton-condensate dispersion relation in a $\mathrm{CuBr}$ microcavity
}

\author{
Masaaki Nakayama and Masafumi Ueda \\ Department of Applied Physics, Graduate School of Engineering, Osaka City University, Sugimoto, Sumiyoshi-ku, Osaka 558-8585, Japan
}

(Received 14 December 2016; revised manuscript received 3 February 2017; published 22 March 2017)

\begin{abstract}
We have investigated the dispersion relation of polariton condensates in a $\mathrm{CuBr}$ microcavity with the use of angle-resolved photoluminescence (PL) spectroscopy at $77 \mathrm{~K}$. The polariton condensation was clearly confirmed by the thresholdlike changes in the PL intensity, energy, and bandwidth of the lower polariton at a zero in-plane wave-vector $k_{\|}=0$ as a function of excitation power density. A blueshifted flat dispersion of the PL energy suddenly appeared at the condensation threshold in a small $k_{\|}$region accompanied by the dispersion of the noncondensate PL as a background. With increasing excitation power density from the threshold, the intensity of the noncondensate PL became negligible. As a result, we found a dispersive profile of the dispersion relation of the condensate in a large $k_{\|}$region in addition to the flat dispersion corresponding to the diffusive profile. The total dispersion relation of the condensate was explained quantitatively by a theoretical model for nonequilibrium condensation.
\end{abstract}

DOI: 10.1103/PhysRevB.95.125315

\section{INTRODUCTION}

In a semiconductor microcavity, spatial confinement of light produces a cavity photon, so that strong coupling between excitons and cavity photons gives rise to exciton polaritons, the so-called cavity polaritons [1]. In the past decade, considerable attention has been paid to Bose-Einstein condensation and polariton lasing from nonequilibrium condensates in microcavities because of the bosonic nature of the cavity polariton [2-18]. The effective mass of the cavity polariton is very light relative to that of a bare exciton: typically on the order of $10^{-4}$. This is a great merit for cavity-polariton condensation because achieving unity for the state occupancy at a zero in-plane wavevector $k_{\|}=0$ is easy even at room temperature owing to the extremely low density of states. From the viewpoint of thermal stability of the cavity polariton, wide-gap semiconductors, such as $\mathrm{GaN}$ and $\mathrm{ZnO}$, were used as an active layer in the microcavities because of the large exciton binding energies: $E_{\mathrm{b}}=26$ (63) $\mathrm{meV}$ for $\mathrm{GaN}(\mathrm{ZnO})$ [19]. Room-temperature condensation was confirmed in $\mathrm{GaN}[5,8,10,11,16]$ and $\mathrm{ZnO}$ microcavities $[15,16]$. The Rabi splitting energy corresponding to the strong-coupling strength is also an important factor for the stability of the cavity polariton in condensation. A large Rabi splitting energy, which largely separates the energies of the lower polariton (LP) and cavity photon at $k_{\|}=0$, prevents the strong coupling from turning to weak coupling in a highdensity excitation regime [20]. We reported that the Rabi splitting energies in $\mathrm{CuBr}$ and $\mathrm{CuCl}$ microcavities are on the order of $100 \mathrm{meV}[18,21,22]$. Note that the exciton binding energies of $\mathrm{CuBr}$ and $\mathrm{CuCl}$ are 108 and $190 \mathrm{meV}$, respectively [19].

The dispersion relation of cavity-polariton condensates is a key issue from an aspect of thermodynamics of condensation; however, there have been limited works on the dispersion relation as follows. In equilibrium condensation, the dispersion relation corresponds to the Bogoliubov mode with an acousticphonon-like dispersion, which was confirmed in GaAs [9] and InGaAs [14] quantum-well (QW) microcavities using angleresolved photoluminescence (PL) [9] and four-wave mixing [14] spectroscopic techniques. In contrast, in nonequilibrium condensation, it was theoretically predicted that the dispersion relation becomes flat in a small $k_{\|}$region [23,24], which is called a diffusive Goldstone mode. It should be noted that the dispersion relation of the Goldstone mode becomes dispersive similar to the Bogoliubov mode in a large $k_{\|}$ region. In Ref. [13], the dispersion relations measured with angle-resolved PL spectroscopy in a GaAs QW microcavity were analyzed using the theory in Ref. [24]; however, it was unclear to differentiate whether the Bogoliubov mode or the diffusive Goldstone mode matches the experimental results. In our previous work with angle-resolved PL spectroscopy [18], we clearly observed a blueshifted flat dispersion in a $\mathrm{CuBr}$ microcavity and suggested the possibility of the diffusive Goldstone mode. We, however, could not observe the dispersive profile in a large $k_{\|}$region because of a large damping rate of polariton condensates. Thus, the dispersion relation in nonequilibrium condensation has not been settled experimentally until now.

In this paper, we report the dispersion relation in the cavity-polariton condensation observed with angle-resolved PL spectroscopy in a $\mathrm{CuBr}$ microcavity. To characterize the intrinsic properties of the cavity polariton, we observed the cavity-polariton dispersion using angle-resolved reflectance spectroscopy and analyzed the dispersion relation with a phenomenological Hamiltonian for the strong coupling. The threshold of the polariton condensation was confirmed by the excitation power dependence of the PL spectrum at $k_{\|}=0$. We found that the dispersion relation of the LP is changed dramatically by the polariton condensation. Below the threshold, the dispersion relation matches the intrinsic LP one. In contrast, above the threshold, the dispersion relation, which is considerably blueshifted by cavity-polariton renormalization, is flat (diffusive) in a small $k_{\|}$region and dispersive in a large $k_{\|}$region. The diffusive and dispersive profiles of the dispersion relation are discussed quantitatively on the basis of the theory for the Goldstone mode in nonequilibrium condensation [24].

\section{EXPERIMENTAL DETAILS}

We used a $\mathrm{CuBr}$ microcavity with $\mathrm{HfO}_{2} / \mathrm{SiO}_{2}$ distributed Bragg reflectors (DBRs) grown on a (0001) $\mathrm{Al}_{2} \mathrm{O}_{3}$ substrate 

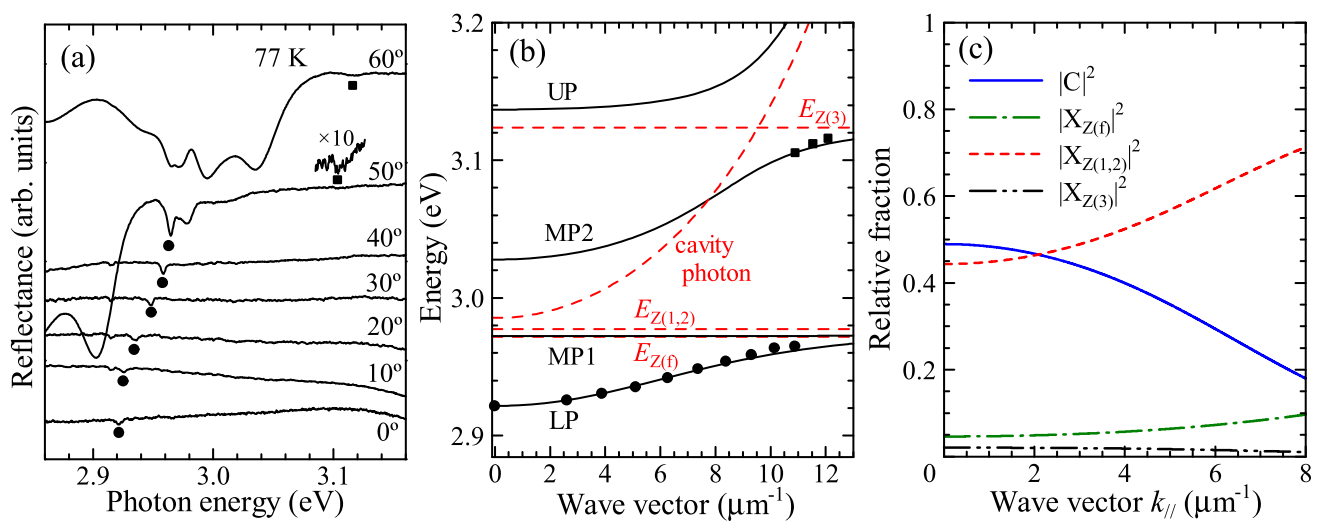

FIG. 1. (a) Angle-resolved reflectance spectra at various incident angles from $\theta=0^{\circ}$ to $60^{\circ}$ at $77 \mathrm{~K}$ in the $\mathrm{CuBr}$ microcavity. (b) Dispersion relations of the cavity polaritons (solid curves) and cavity photon (dashed curve) estimated from analysis of the incident angle dependence of reflectance dip energies (solid circles and squares) with a phenomenological Hamiltonian for the strong coupling. The horizontal dashed lines depict the energies of the $Z_{\mathrm{f}}, Z_{1,2}$, and $Z_{3}$ excitons. (c) Relative fractions of the $Z_{\mathrm{f}}, Z_{1,2}$, and $Z_{3}$ excitons and cavity photon in the LP as a function of $k_{\|}$

as a sample. The $\mathrm{CuBr}$ active layer and DBRs were prepared with vacuum deposition at $60^{\circ} \mathrm{C}$ and $\mathrm{rf}$ magnetron sputtering at room temperature, respectively. In the vacuum deposition, the source material was $\mathrm{CuBr}$ powders with $99.999 \%$ purity, and the background pressure was $5 \times 10^{-6} \mathrm{~Pa}$. The thickness of the $\mathrm{CuBr}$ active layer was set to $\lambda=\lambda_{\mathrm{EX}} / \sqrt{\varepsilon_{\mathrm{b}}}[25]$, where $\lambda_{\mathrm{EX}}$ is the resonant wavelength of the lowest-lying exciton and $\varepsilon_{\mathrm{b}}$ is the background dielectric constant: $\lambda=208 \mathrm{~nm}$ for $\lambda_{\mathrm{EX}}=$ $418.5 \mathrm{~nm}$ and $\varepsilon_{\mathrm{b}}=4.062 \mathrm{in} \mathrm{CuBr}$ [19]. The bottom and top DBRs, which were terminated by a $\mathrm{HfO}_{2}$ layer, consisted of 15.5 and 12.5 periods, respectively. The source materials in the sputtering process were commercially supplied plates of $\mathrm{HfO}_{2}$ with $99.9 \%$ purity and $\mathrm{SiO}_{2}$ with $99.99 \%$ purity. The sputtering gas was Ar under a pressure of 0.5 Pa. The growth rates during the deposition process were monitored using a crystal oscillator. We confirmed from $\mathrm{x}$-ray diffraction patterns that the $\mathrm{CuBr}$ layer is a crystalline film oriented along the [111] direction.

In PL measurements, the excitation light source was the third-harmonic-generation light $(355 \mathrm{~nm})$ of a laser-diodepumped pulsed yttrium aluminum garnet laser with a pulse duration of $1.0 \mathrm{~ns}$ and a repetition rate of $10 \mathrm{kHz}$. The excitation power was corrected with the transmittance 0.62 of the top DBR at $355 \mathrm{~nm}$ which is away from the wavelength region of the stop band of the DBR. In measuring angle-resolved PL spectra, the excitation light incidence was fixed at $0^{\circ}$, whereas the detection angle was changed with a goniometer. The resolution of the detection angle was $\sim 1^{\circ}$. The excitation light was focused on the sample surface with a spot diameter of $\sim 40 \mu \mathrm{m}$. The PL spectrum was detected using a cooled charge-coupled device attached to a single monochromator with a resolution of $0.1 \mathrm{~nm}$. In addition, angle-resolved reflectance spectra were measured to characterize the intrinsic cavity-polariton dispersions. The probe light source was a Xe lamp, and the reflected light was detected with the same system as that used in the PL measurement. The optical measurements were performed at $77 \mathrm{~K}$ using a liquid-nitrogen cryostat to reduce sample vibrations which disturb the accuracy of angle-resolved PL spectroscopy.

\section{RESULTS AND DISCUSSION}

To discuss the cavity-polariton condensation, it is essential to characterize the intrinsic cavity-polariton properties, such as the dispersion relations, Rabi splitting energies, relative fractions of the exciton and cavity photon, and detuning corresponding to the energy difference between the lowestlying exciton and the cavity photon at $k_{\|}=0$. The excitonic system in a $\mathrm{CuBr}$ crystal with a zinc-blende structure consists of three excitons labeled $Z_{\mathrm{f}}, Z_{1,2}$, and $Z_{3}$ at the $\Gamma$ point. The $Z_{\mathrm{f}}$ exciton corresponds to a triplet exciton; however, its oscillator strength is considerably enhanced by mixing between the triplet and the singlet excitons [26]. The $Z_{1,2}\left(Z_{3}\right)$ exciton is assigned to the degenerate heavy-hole and lighthole excitons (split-off-hole exciton). Therefore, the strong coupling among the three excitons and cavity photon produces four cavity polaritons: the LP, middle polariton 1 (MP1), MP2, and upper polariton (UP) [22]. Figure 1(a) shows the angle-resolved reflectance spectra at various incident angles from $\theta=0^{\circ}$ to $60^{\circ}$ in the $\mathrm{CuBr}$ microcavity. We observed two reflectance dips depending on the incident angle: The two dips are indicated by the solid circle and square. At $\theta=50^{\circ}$ and $60^{\circ}$, the low-energy edge of the stop band appears in the reflectance spectra. The lowest-lying reflectance dip is assigned to the LP. The apparent quality factor was estimated to be $Q_{\mathrm{a}}=E_{\mathrm{LP}} / \Delta E_{\text {dip }}=7.1 \times 10^{2}$, where $E_{\mathrm{LP}}$ and $\Delta E_{\mathrm{dip}}$ are the LP energy and full width at half maximum (FWHM) of the reflectance dip at $\theta=0^{\circ}$, respectively.

Figure 1(b) shows the dispersion relations of the cavity polaritons (solid curves) and cavity photon (dashed curve) estimated from analysis of the incident angle dependence of the observed reflectance dip energies indicated by the solid circle and square. Here, we converted $\theta$ to $k_{\|}$using the following equation [27]: $k_{\|}=E_{\mathrm{ph}}(0) \sin \theta /\left(\hbar c \sqrt{1-\sin ^{2} \theta / n_{\mathrm{eff}}^{2}}\right)$, where $E_{\mathrm{ph}}(0)$ is the cavity-photon energy at $k_{\|}=0, n_{\text {eff }}$ is the effective refractive index of the cavity, and $c$ is the speed of light in vacuum. The values of $E_{\mathrm{ph}}(0)$ and $n_{\mathrm{eff}}$ are $2.985 \mathrm{eV}$ and 2.24 , respectively, which were estimated from analysis of the cavity-polariton and cavity-photon dispersion relations. 
The horizontal dashed lines depict the energies of the $Z_{\mathrm{f}}, Z_{1,2}$, and $Z_{3}$ excitons obtained from the absorption spectrum of a $\mathrm{CuBr}$ thin film (not shown here). The analysis of the cavity-polariton and cavity-photon dispersion relations was based on a phenomenological Hamiltonian for the strong coupling among the three excitons and the cavity photon. The details of the analysis method were described in Ref. [22]. The observed reflectance dips indicated by the solid circle and square are clearly attributed to the LP and MP2, respectively. The intensity of the MP1 reflectance signal is usually very weak [22]; therefore, it could not be observed in this sample. The reason why the UP reflectance signal was not observed is that the strong absorption by the exciton continuum state, the energy region of which overlaps with that of the UP, prevents the appearance of the UP signal [28]. The Rabi splitting energies obtained from the analysis are 31,106 , and $83 \mathrm{meV}$ for the $Z_{\mathrm{f}}, Z_{1,2}$, and $Z_{3}$ excitons, respectively, and the detuning is $+14 \mathrm{meV}$.

Here, we estimate the LP lifetime $\tau_{\mathrm{LP}}$ at $k_{\|}=0$, which is important in the discussion of the cavity-polariton condensation. The LP lifetime is given by [18]

$$
\begin{aligned}
1 / \tau_{\mathrm{LP}}= & \left|X_{Z(\mathrm{f})}\right|^{2} / \tau_{Z(\mathrm{f})}+\left|X_{Z(1,2)}\right|^{2} / \tau_{Z(1,2)} \\
& +\left|X_{Z(3)}\right|^{2} / \tau_{Z(3)}+|C|^{2} / \tau_{\mathrm{ph}},
\end{aligned}
$$

where $\left|X_{Z(\mathrm{f})}\right|^{2}\left(\tau_{Z(\mathrm{f})}\right),\left|X_{Z(1,2)}\right|^{2}\left(\tau_{Z(1,2)}\right)$, and $\left|X_{Z(3)}\right|^{2}\left(\tau_{Z(3)}\right)$ are the relative fractions (lifetimes) of the $Z_{\mathrm{f}}, Z_{1,2}$, and $Z_{3}$ excitons, respectively, and $|C|^{2}$ and $\tau_{\mathrm{ph}}$ are the relative fraction and lifetime of the cavity photon, respectively. The relative fraction, which is also called the Hopfield coefficient, corresponds to the square of the eigenvector of the phenomenological Hamiltonian used in the analysis of the cavity-polariton and cavity-photon dispersion relations. Figure 1(c) shows the relative fractions of the $Z_{\mathrm{f}}, Z_{1,2}$, and $Z_{3}$ excitons and cavity photon in the LP as a function of $k_{\|}$. The values of $\left|X_{Z(\mathrm{f})}\right|^{2},\left|X_{Z(1,2)}\right|^{2},\left|X_{Z(3)}\right|^{2}$, and $|C|^{2}$ at $k_{\|}=0$ are $0.05,0.44$, 0.02 , and 0.49 , respectively. The major relative fractions are $\left|X_{Z(1,2)}\right|^{2}$ and $|C|^{2}$. The cavity-photon lifetime is given by $\tau_{\mathrm{ph}}=Q /\left[2 \pi E_{\mathrm{ph}}(0) / h\right]$ [29]. Here, we have to correct the apparent quality factor $Q_{\mathrm{a}}$ using the relative fraction of the cavity photon: $Q=Q_{\mathrm{a}} /|C|^{2}=1.4 \times 10^{3}$. Thus, the cavityphoton lifetime is calculated to be $0.31 \mathrm{ps}$. The lifetime of the $Z_{1,2}$ exciton, which is the major exciton component of the LP, was reported to be $150 \mathrm{ps}$ [30], which is much longer than $\tau_{\mathrm{ph}}$. As a result, the LP lifetime is approximately calculated to be $\tau_{\mathrm{LP}} \approx \tau_{\mathrm{ph}} /|C|^{2}=0.63 \mathrm{ps}$. This very short lifetime suggests the occurrence of nonequilibrium cavity-polariton condensation because the rapid decay prevents the equilibrium accumulation of condensates in the relaxation process.

In the following, we describe the results and discussion for the cavity-polariton condensation. Figure 2 shows the average excitation power-density dependence of the PL spectrum at $k_{\|}=0$ at $77 \mathrm{~K}$, where the vertical dashed line indicates the LP energy at $k_{\|}=0$ obtained from the reflectance spectrum at $\theta=0^{\circ}$. At the lowest excitation density of $0.5 \mathrm{~W} / \mathrm{cm}^{2}$, the PL peak energy just agrees with the LP energy. The LP-PL band exhibits a blueshift, which reflects the cavity-polariton renormalization, with increasing excitation power density. At $4.0 \mathrm{~W} / \mathrm{cm}^{2}$, the sharp PL band labeled $\mathrm{B}_{\text {th }}$ appears with a thresholdlike nature at the high-energy side of the LP-PL

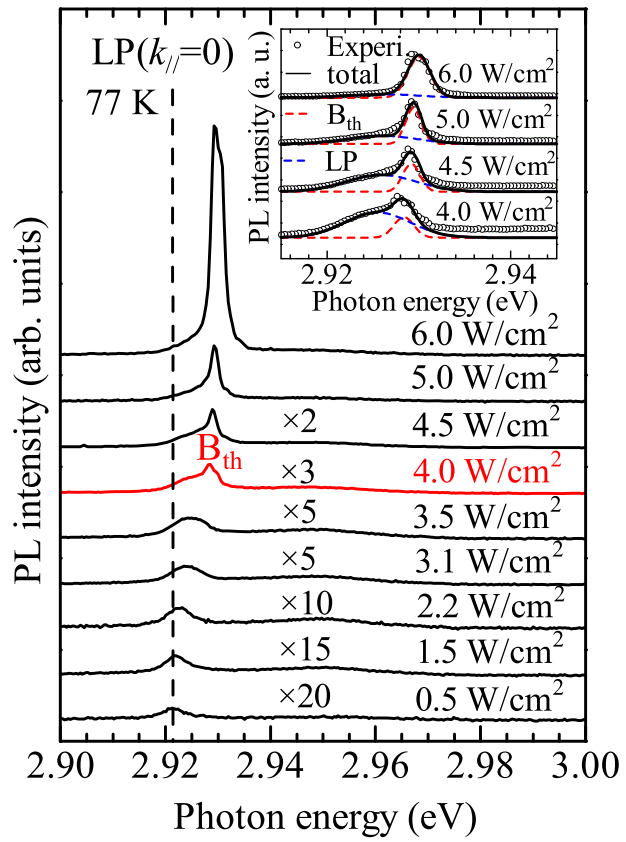

FIG. 2. Average excitation power-density dependence of the PL spectrum at $k_{\|}=0$ at $77 \mathrm{~K}$, where the vertical dashed line indicates the LP energy at $k_{\|}=0$ obtained from the reflectance spectrum at $\theta=0^{\circ}$. The inset shows the results of the line-shape analysis of the PL spectra (open circles) above the threshold for the $\mathrm{B}_{\mathrm{th}}-\mathrm{PL}$ band, where the PL intensity is normalized to the maximum value at each excitation power density. The dashed curves indicate the results of decomposition of the PL spectrum with two Gaussian functions corresponding to the $\mathrm{B}_{\mathrm{th}}-\mathrm{PL}$ and LP-PL bands, and the solid curve depicts the sum of the decomposed two bands.

band. The inset of Fig. 2 shows the results of line-shape analysis of the PL spectra (open circles) above the threshold for the $\mathrm{B}_{\mathrm{th}}-\mathrm{PL}$ band, where the PL intensity is normalized to the maximum value at each excitation power density. The dashed curves indicate the results of decomposition of the PL spectrum with two Gaussian functions corresponding to the $\mathrm{B}_{\text {th }}$-PL and LP-PL bands, and the solid curve depicts the sum of the decomposed two bands. The line-shape analysis well reproduces the PL spectra. The weak background in the higher-energy side of the $\mathrm{B}_{\mathrm{th}} \mathrm{PL}$ band can be attributed to the PL from donor-acceptor pair recombination [31]. It is evident from the inset of Fig. 2 that the $B_{t h}-P L$ band markedly grows relative to the LP-PL band with an increase in excitation power density from the threshold. Figures 3(a)-3(c) show the integrated intensity, FWHM, and peak energy of the LP-PL and $\mathrm{B}_{\mathrm{th}}-\mathrm{PL}$ bands at $k_{\|}=0$, respectively, as a function of average excitation power density. Note that the $\mathrm{CuBr}$ active layer was sometimes damaged by a further increase in excitation power density from $6.0 \mathrm{~W} / \mathrm{cm}^{2}$, so that the highest excitation power density was fixed at $6.0 \mathrm{~W} / \mathrm{cm}^{2}$ to obtain reproducible experimental data. It is obvious that the thresholdlike changes in the three PL parameters simultaneously occur at $4.0 \mathrm{~W} / \mathrm{cm}^{2}$, which is indicated by the vertical dashed line. This fact demonstrates the occurrence of the cavity-polariton condensation. The coexistence of the $\mathrm{B}_{\text {th }}-\mathrm{PL}$ and LP-PL bands above the condensation threshold 

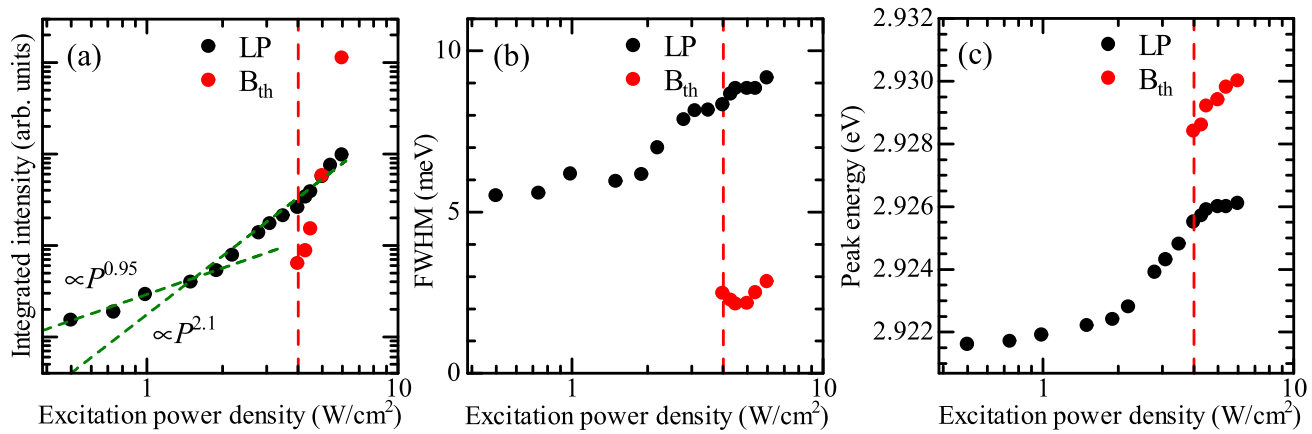

FIG. 3. (a) Integrated intensity, (b) FWHM, and (c) peak energy of the LP-PL and $\mathrm{B}_{\mathrm{th}}$-PL bands at $k_{\|}=0$ as a function of average excitation power density. The green dashed lines in (a) indicate the fitted results of the excitation power-density dependence of the integrated intensity of the LP-PL band.

reflects spatial inhomogeneity of the sample quality and/or that of excitation power density. In Fig. 3(a), the dashed lines indicate the fitted results of the excitation power-density dependence of the integrated intensity of the LP-PL band. The almost square dependence is observed above $\sim 2 \mathrm{~W} / \mathrm{cm}^{2}$. This suggests that polariton-polariton scattering dominates the relaxation process in momentum space. The width broadening and peak-energy blueshift, which are due to many-body effects on the cavity polaritons, also become remarkable above $\sim 2 \mathrm{~W} / \mathrm{cm}^{2}$ as shown in Figs. 3(b) and 3(c). Thus, polaritonpolariton scattering causes the considerable accumulation of LPs at $k_{\|}=0$ in this case. The peak energy of the $\mathrm{B}_{\text {th }}-\mathrm{PL}$ band, $2.9300 \mathrm{eV}$ at the highest excitation power density of $6.0 \mathrm{~W} / \mathrm{cm}^{2}$ is sufficiently below the cavity-photon energy at $k_{\|}=0: E_{\mathrm{ph}}(0)=2.985 \mathrm{eV}$. In addition, the blueshift energy of the $\mathrm{B}_{\mathrm{th}}-\mathrm{PL}$ band at $6.0 \mathrm{~W} / \mathrm{cm}^{2}, 8.6 \mathrm{meV}$, relative to the intrinsic LP energy at $k_{\|}=0$ is considerably smaller than the Rabi splitting energies of 31 and $106 \mathrm{meV}$ for the $Z_{\mathrm{f}}$ and $Z_{1,2}$ excitons. These facts verify that the strong-coupling regime is fully stable in the cavity-polariton condensation. According to Ref. [32], a decrease in the exciton binding energy $E_{\mathrm{b}}$ and oscillator strength $f_{\mathrm{X}}$ due to Coulomb interactions is the major factor for the blueshift in a wide-gap semiconductor with a large $E_{\mathrm{b}}$. The magnitude of the $E_{\mathrm{b}}$ decrease is proportional to $E_{\mathrm{b}}$, and the Rabi splitting energy is proportional to the square root of $f_{\mathrm{X}}$ [32]. Thus, the large exciton binding energy and Rabi splitting energies in the $\mathrm{CuBr}$ microcavity result in the large blueshift in comparison with GaAs QW microcavities: typically $1 \mathrm{meV}$ around a condensation threshold $[4,9,13]$. Note that the blueshift mechanism will be taken into account in the analysis of the polariton-condensate dispersion relation below.

Here, we focus on the excitation power-density dependence of the LP dispersion relation. Figure 4 shows the image maps of angle-resolved PL spectra at $77 \mathrm{~K}$ as a function of $k_{\|}$at various average excitation power densities of (a) 0.5 , (b) 3.5 , (c) 4.0 , and (d) $6.0 \mathrm{~W} / \mathrm{cm}^{2}$, where the PL intensity, which is normalized to the maximum intensity at each excitation power density, is indicated by the color scale. The black dashed curve depicts the intrinsic LP dispersion relation taken from Fig. 1(b). At the lowest excitation power density of $0.5 \mathrm{~W} / \mathrm{cm}^{2}$, the PL energy as a function of $k_{\|}$agrees with the intrinsic LP dispersion relation. The PL intensity around $k_{\|}=0$ is the strongest. At $3.5 \mathrm{~W} / \mathrm{cm}^{2}$, which is slightly lower than the condensation threshold of $4.0 \mathrm{~W} / \mathrm{cm}^{2}$, the PL energy as a function of $k_{\|}$exhibits a parallel blueshift relative to the intrinsic LP dispersion relation. The LP-PL intensity is almost uniform in the whole $k_{\|}$region in Fig. 4 (b) at $3.5 \mathrm{~W} / \mathrm{cm}^{2}$, which is in contrast to Fig. 4 (a) at $0.5 \mathrm{~W} / \mathrm{cm}^{2}$. It is evident from Fig. 3(a) that the excitation power-density dependence of the LP-PL intensity is classified into two categories: the linear and the square dependences below and above
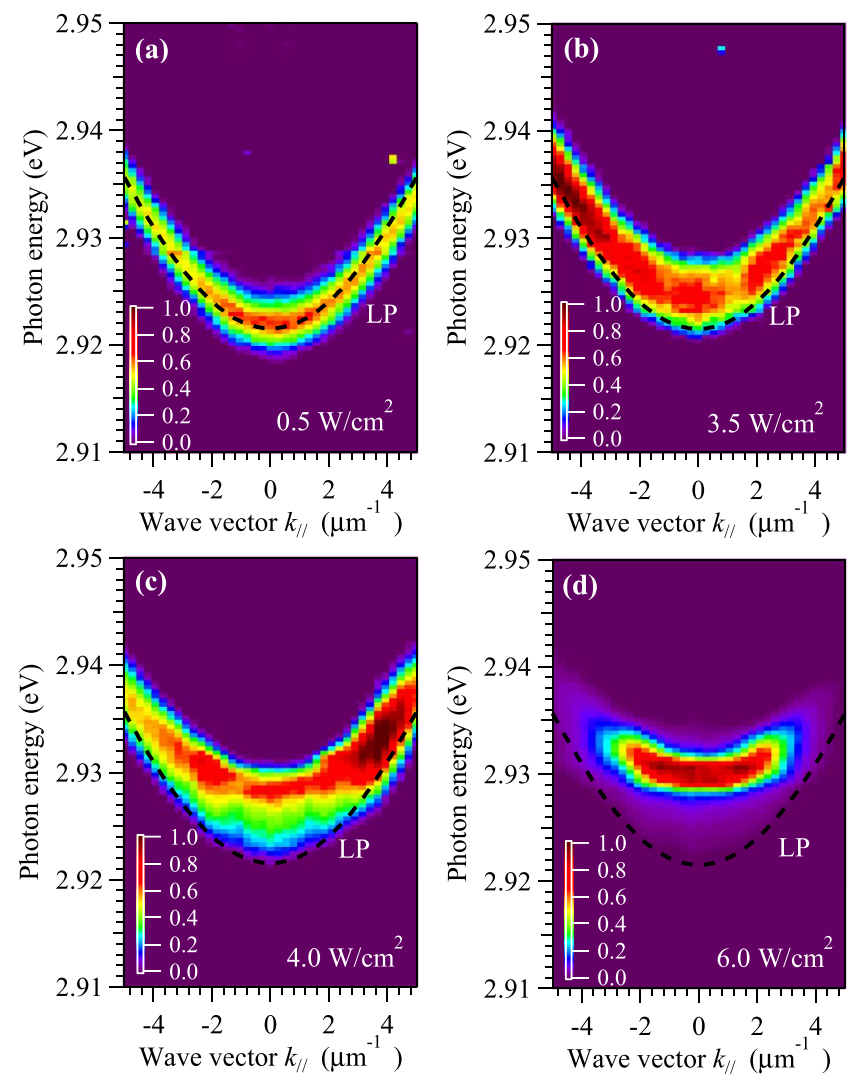

FIG. 4. Image maps of angle-resolved PL spectra at $77 \mathrm{~K}$ as a function of $k_{\|}$at various average excitation power densities of (a) 0.5 , (b) 3.5 , (c) 4.0 , and (d) $6.0 \mathrm{~W} / \mathrm{cm}^{2}$, where the PL intensity, which is normalized to the maximum intensity at each excitation power density, is indicated by the color scale. The black dashed curve depicts the intrinsic LP dispersion relation taken from Fig. 1(b). 
$\sim 2 \mathrm{~W} / \mathrm{cm}^{2}$, respectively. In the linear dependence, polaritonphonon scattering is dominant in the relaxation process; therefore, thermalization of polaritons leads to the fact that the LP-PL intensity around $k_{\|}=0$ is the strongest as shown in Fig. 4(a), which is a usual phenomenon. In contrast, the square dependence indicates that polariton-polariton scattering is dominant in the relaxation process. Thus, a possible origin of the almost uniform distribution of the LP-PL intensity shown in Fig. 4(b) can be phenomenologically attributed to the phenomenon that the polariton-polariton scattering process produces photon emission during the relaxation in the LP dispersion. At the threshold of $4.0 \mathrm{~W} / \mathrm{cm}^{2}$, the blueshifted flat dispersion of the PL energy suddenly appears in the $k_{\|}$ region less than $\sim 1.2 \mu \mathrm{m}^{-1}$ in addition to the noncondensate LP-PL dispersion. At $6.0 \mathrm{~W} / \mathrm{cm}^{2}$, the noncondensate LP-PL dispersion is negligible, which is also obvious in the PL spectrum at $k_{\|}=0$ shown in Fig. 2. Thus, the observed PL-energy dispersion originates from the cavity-polariton condensation. It should be noted that the dispersion relation consists of two parts: dispersionless in the $k_{\|}$region smaller than $\sim 1.0 \mu \mathrm{m}^{-1}$ and dispersive in the larger $k_{\|}$region. This is a key finding in this work. In our previous work [18], we observed only the blueshifted flat dispersion. In the previous sample, the detuning was $-22 \mathrm{meV}$, whereas that is $+14 \mathrm{meV}$ in the present sample. The $Q$ values in the two samples are almost the same. It was reported that the thermodynamics of cavity-polariton condensation is affected by the detuning $[11,16]$. Thus, the difference of the detuning values in the present and previous samples may result in the difference of the observed dispersions. The further discussion for the detuning effect is beyond the scope of this paper.

To analyze the observed dispersion relation shown in Fig. 4(d), we used a theoretical model for nonequilibrium condensation, the so-called Goldstone mode, reported in Ref. [24]. The dispersion relation of the Goldstone mode derived from the Gross-Pitaevskii equation is given by [24]

$$
\begin{aligned}
E_{\mathrm{G}}\left(k_{\|}\right)= & E_{\mathrm{B}}(0)-i \frac{\hbar(2 \pi \Gamma)}{2} \\
& +\sqrt{\left[E_{\mathrm{B}}\left(k_{\|}\right)-E_{\mathrm{B}}(0)\right]^{2}-\frac{\hbar^{2}(2 \pi \Gamma)^{2}}{4}},
\end{aligned}
$$

where $E_{\mathrm{B}}\left(k_{\|}\right)$is the Bogoliubov-mode dispersion relation in equilibrium condensation and $\Gamma$ is the effective relaxation rate of the Bogoliubov mode, namely, $\Gamma$ reflects the nonequilibrium of the system. The Bogoliubov-mode dispersion relation is written as

$$
E_{\mathrm{B}}\left(k_{\|}\right)=E_{\mathrm{B}}(0)+\sqrt{\frac{\hbar^{2} k_{\|}^{2}}{2 M_{\mathrm{LP}}}\left(\frac{\hbar^{2} k_{\|}^{2}}{2 M_{\mathrm{LP}}}+2 \hbar \eta\right)},
$$

where $M_{\mathrm{LP}}$ is the effective mass of the LP and $\hbar \eta$ is a polariton-polariton interaction energy within condensates. The real part of Eq. (2) gives the dispersion relation. It is evident from Eq. (2) that the dispersion relation is flat (diffusive) in $\left[E_{\mathrm{B}}\left(k_{\|}\right)-E_{\mathrm{B}}(0)\right]^{2}-\hbar^{2}(2 \pi \Gamma)^{2} / 4 \leqslant 0$ : the diffusive Goldstone mode in a small $k_{\|}$region. In $\left[E_{\mathrm{B}}\left(k_{\|}\right)-E_{\mathrm{B}}(0)\right]^{2}-$ $\hbar^{2}(2 \pi \Gamma)^{2} / 4>0$, the dispersion relation exhibits a dispersive profile approaching $E_{\mathrm{B}}\left(k_{\|}\right)$in a large $k_{\|}$region. Note that the simultaneous observation of the diffusive and dispersive pro-

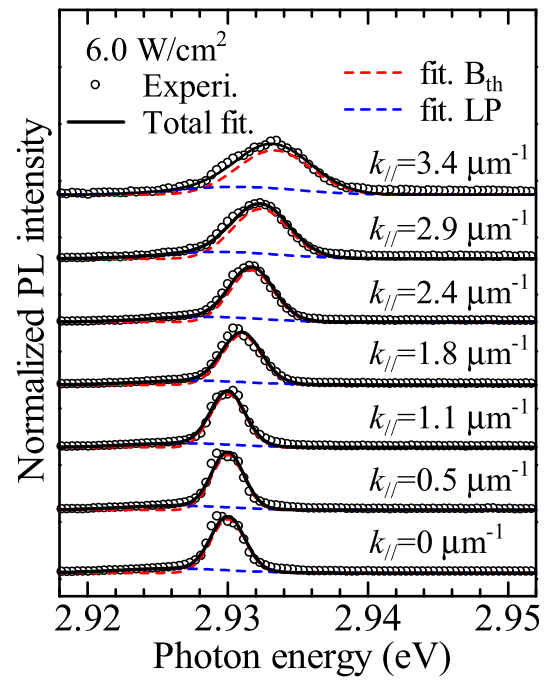

FIG. 5. PL spectra (open circles) at various $k_{\|}$values taken from Fig. 4(d) at $6.0 \mathrm{~W} / \mathrm{cm}^{2}$, where the PL intensity is normalized to the maximum value at each $k_{\|}$. The dashed curves indicate the results of decomposition of the PL spectrum with two Gaussian functions corresponding to the $\mathrm{B}_{\mathrm{th}}-\mathrm{PL}$ and LP-PL bands, and the solid curve depicts the sum of the decomposed two bands.

files of the dispersion relation in nonequilibrium condensation is experimentally reported here.

Finally, we quantitatively discuss the dispersion relation in the cavity-polariton condensation. Figure 5 shows the PL spectra (open circles) at various $k_{\|}$values taken from Fig. 4(d) at $6.0 \mathrm{~W} / \mathrm{cm}^{2}$, where the PL intensity is normalized to the maximum value at each $k_{\|}$. The dashed curves indicate the results of decomposition of the PL spectrum with two Gaussian functions corresponding to the $\mathrm{B}_{\mathrm{th}}-\mathrm{PL}$ and LP-PL bands, and the solid curve depicts the sum of the decomposed two bands. It is evident that the line-shape analysis well reproduces the PL spectra. Note that the $\mathrm{B}_{\mathrm{th}}$-PL and LP-PL bands originate from the LP condensate and noncondensate, respectively. The coexistence of the $\mathrm{B}_{\mathrm{th}}-\mathrm{PL}$ and LP-PL bands is clearly observed from the excitation power-density dependence of the PL spectrum at $k_{\|}=0$ shown in Fig. 2. Figure 6 shows the peak energies of the $\mathrm{B}_{\mathrm{th}}$-PL and LP-PL bands (solid and open circles) obtained from the above line-shape analysis of the PL spectra taken from Fig. 4(d) as a function of $k_{\|}$, where the green solid line indicates the fitted dispersion relation of the LP condensate using Eq. (2), the red dashed line depicts the Bogoliubov-mode dispersion relation given by Eq. (3), and the black solid curve represents the intrinsic LP dispersion relation taken from Fig. 1(b) for reference. The errors of the $\mathrm{B}_{\mathrm{th}}-\mathrm{PL}$ and LP-PL peak energies estimated from the line-shape analysis are within \pm 0.2 and $\pm 0.3 \mathrm{meV}$, respectively. The black dashed curve indicates the result of parabolic fitting of the $k_{\|}$dependence of the LP-PL peak energy. It is evident that the dispersion relation of the LP condensate apparently separates from the intrinsic and blueshifted LP dispersion relations.

According to Ref. [9], $\hbar \eta$ for the Bogoliubov mode in Eq. (3) corresponds to the blueshift energy of condensates at 


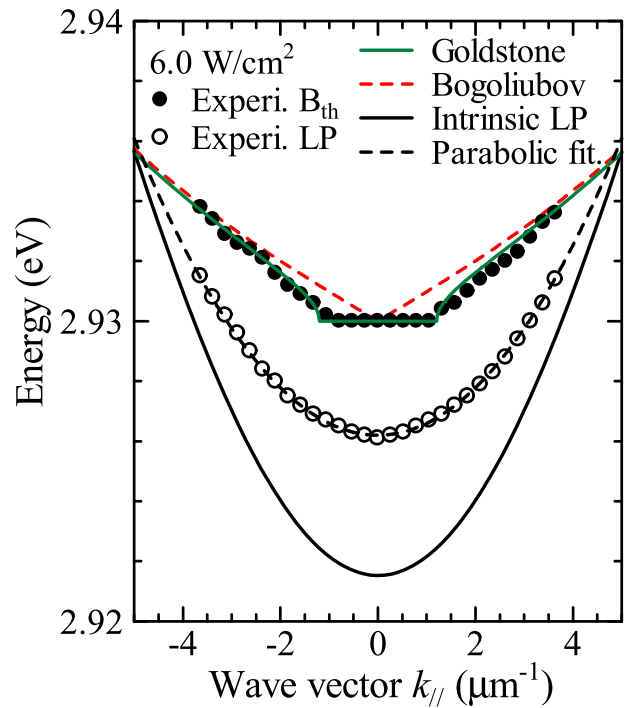

FIG. 6. Peak energies of the $\mathrm{B}_{\text {th }}$-PL and LP-PL bands (solid and open circles) obtained from the line-shape analysis, which is the same manner as that in Fig. 5, of the PL spectra taken from Fig. 4(d) at $6.0 \mathrm{~W} / \mathrm{cm}^{2}$ as a function of $k_{\|}$, where the green solid line indicates the fitted dispersion relation of the LP condensate using Eq. (2), the red dashed line depicts the Bogoliubov-mode dispersion relation given by Eq. (3), and the black solid curve represents the intrinsic LP dispersion relation taken from Fig. 1(b) for reference. The black dashed curve indicates the result of parabolic fitting of the $k_{\|}$dependence of the LP-PL peak energy.

$k_{\|}=0: E_{\mathrm{B}}(0)-E_{\mathrm{LP}}(0)$, where $E_{\mathrm{LP}}(0)$ is the noncondensate LP energy. As described above, the blueshift of the noncondensate LP-PL energy is mainly due to Coulomb interaction effects on $E_{\mathrm{b}}$ and $f_{\mathrm{X}}$, which is not related to the polariton-polariton interaction. We, therefore, adopt the highest PL energy of the noncondensate $\mathrm{LP}$ at $k_{\|}=0$ as $E_{\mathrm{LP}}(0): 2.9261 \mathrm{eV}$ at $6.0 \mathrm{~W} / \mathrm{cm}^{2}$. The peak energy of the $\mathrm{B}_{\mathrm{th}}-\mathrm{PL}$ band at $k_{\|}=0$ at $6.0 \mathrm{~W} / \mathrm{cm}^{2}$ corresponds to $E_{\mathrm{B}}(0): 2.9300 \mathrm{eV}$. Thus, we fixed $\hbar \eta$ at $3.9 \mathrm{meV}$ in the fitting process. The values of the adjustable parameters are as follows: $\Gamma=5.7 \times 10^{11} \mathrm{~s}^{-1}$ and $M_{\mathrm{LP}}=3.1 \times 10^{-4} m_{0}=4.5 M_{\mathrm{LP} 0}$, where $m_{0}$ is the free electron mass and $M_{\mathrm{LP} 0}$ is the effective mass of the intrinsic LP at $k_{\|}=0$ given by $\hbar^{2} /\left[d^{2} E_{\mathrm{LP}}\left(k_{\|}\right) / d k_{\|}^{2}\right]$. It is obvious that the fitted result of the Goldstone-mode dispersion relation totally agrees with the diffusive and dispersive profiles of the experimental dispersion relation. The cut-off wave vector of the flat dispersion is $1.0 \mu \mathrm{m}^{-1}$. In the theoretical framework of Ref. [24], $\Gamma$ should be smaller than the loss rate of the cavity polariton. There are two possible assumptions for the estimation of the loss rate. One is the inverse of the LP lifetime estimated above $1 / \tau_{\mathrm{LP}}=1.6 \times 10^{12} \mathrm{~s}^{-1}$, and the other is based on the energy-time uncertainty principle
$\Delta t \approx \hbar / \Delta E$ [33], where $\Delta E$ and $\Delta t$ correspond to the FWHM of the $\mathrm{B}_{\mathrm{th}}$-PL band and cavity-polariton lifetime, respectively: $1 / \Delta t \approx 4.3 \times 10^{12} \mathrm{~s}^{-1}$ for $\Delta E=2.8 \mathrm{meV}$ at $6.0 \mathrm{~W} / \mathrm{cm}^{2}$. Thus, it is evident that the value of $\Gamma$ is sufficiently smaller than the estimated loss rates, which verifies the validity of the fitting of the dispersion relation. We qualitatively discuss the effective mass value of $4.5 M_{\mathrm{LP} 0}$ estimated from the analysis of the condensate dispersion relation. Note that there are no available theoretical and experimental reports for the effect of cavity-polariton renormalization due to a blueshift on the effective mass. Here, we focus on the blueshifted LP dispersion relation indicated by the open circle in Fig. 6 . The effective mass was estimated to be $1.5 M_{\mathrm{LP} 0}$ using parabolic fitting of the dispersion relation indicated by the black dashed curve. This estimation indicates that the blueshift-induced cavity-polariton renormalization results in a heavier effective mass. As shown in Fig. 6, the blueshift of the dispersion relation of the condensate is about two times larger than that of the LP; therefore, it is expected that the condensate effective mass becomes heavier than $1.5 M_{\mathrm{LP} 0}$. The quantitative discussion requires a theory for the cavity-polariton renormalization effect on the effective mass, which is beyond the scope of this paper.

\section{CONCLUSIONS}

We have investigated the excitation power dependence of the dispersion relation of the LP at $77 \mathrm{~K}$ using angleresolved PL spectroscopy from the viewpoint of the cavitypolariton condensation. We confirmed the cavity-polariton condensation from the fact that the thresholdlike changes in the PL intensity, FWHM, and peak energy at $k_{\|}=0$ simultaneously occur at an average excitation power density of $4.0 \mathrm{~W} / \mathrm{cm}^{2}$. At the condensation threshold, the blueshifted flat dispersion suddenly appears in the small $k_{\|}$region less than $\sim 1.2 \mu \mathrm{m}^{-1}$ in addition to the noncondensate LP dispersion as a background. At the highest excitation power density of $6.0 \mathrm{~W} / \mathrm{cm}^{2}$, the noncondensate LP-PL becomes negligible, so that the dispersion relation in the cavity-polariton condensation is clarified. We found that the dispersion relation consists of two parts: dispersionless (diffusive) in the $k_{\|}$ region smaller than $1.0 \mu \mathrm{m}^{-1}$ and dispersive in the larger $k_{\|}$region. We demonstrated that the observed diffusive and dispersive profiles of the dispersion relation are reasonably explained by the theoretical model for the Goldstone mode in nonequilibrium condensation.

\section{ACKNOWLEDGMENTS}

This work was supported by KAKENHI Grant No. 15H03678 from the Japan Society for the Promotion of Science. We are grateful to D. Kim for his help in the sample preparation.
[1] A. V. Kavokin, J. J. Baumberg, G. Malpuech, and F. P. Laussy, Microcavities (Oxford University Press, Oxford, 2007), p. 117.

[2] M. Richard, J. Kasprzak, R. André, R. Romestain, L. S. Dang, G. Malpuech, and A. Kavokin, Phys. Rev. B 72, 201301 (2005).
[3] J. Kasprzak, M. Richard, S. Kundermann, A. Baas, P. Jeambrun, J. M. J. Keeling, F. M. Marchetti, M. H. Szymańska, R. André, J. L. Staehli, V. Savona, P. B. Littlewood, B. Deveaud, and L. S. Dang, Nature (London) 443, 409 (2006). 
[4] R. Balili, V. Hartwell, D. Snoke, L. Pfeiffer, and K. West, Science 316, 1007 (2007).

[5] S. Christopoulos, G. Baldassarri Höger von Högersthal, A. J. D. Grundy, P. G. Lagoudakis, A. V. Kavokin, J. J. Baumberg, G. Christmann, R. Butté, E. Feltin, J.-F. Carlin, and N. Grandjean, Phys. Rev. Lett. 98, 126405 (2007).

[6] A. P. D. Love, D. N. Krizhanovskii, D. M. Whittaker, R. Bouchekioua, D. Sanvitto, S. A. Rizeiqi, R. Bradley, M. S. Skolnick, P. R. Eastham, R. André, and L. S. Dang, Phys. Rev. Lett. 101, 067404 (2008).

[7] J. Kasprzak, D. D. Solnyshkov, R. André, L. S. Dang, and G. Malpuech, Phys. Rev. Lett. 101, 146404 (2008).

[8] G. Christmann, R. Butté, E. Feltin, J.-F. Carlin, and N. Grandjean, Appl. Phys. Lett. 93, 051102 (2008).

[9] S. Utsunomiya, L. Tian, G. Roumpos, C. W. Lai, N. Kumada, T. Fujisawa, M. Kuwata-Gonokami, A. Löffler, S. Höfling, A. Forchel, and Y. Yamamoto, Nat. Phys. 4, 700 (2008).

[10] J. J. Baumberg, A. V. Kavokin, S. Christopoulos, A. J. D. Grundy, R. Butté, G. Christmann, D. D. Solnyshkov, G. Malpuech, G. Baldassarri Höger von Högersthal, E. Feltin, J.-F. Carlin, and N. Grandjean, Phys. Rev. Lett. 101, 136409 (2008).

[11] J. Levrat, R. Butté, E. Feltin, J.-F. Carlin, N. Grandjean, D. Solnyshkov, and G. Malpuech, Phys. Rev. B 81, 125305 (2010).

[12] T. Guillet, M. Mexis, J. Levrat, G. Rossbach, C. Brimont, T. Bretagnon, B. Gil, R. Butté, N. Grandjean, L. Orosz, F. Réveret, J. Leymarie, J. Zúñiga-Pérez, M. Leroux, F. Semond, and S. Bouchoule, Appl. Phys. Lett. 99, 161104 (2011).

[13] M. Aßmann, J.-S. Tempel, F. Veit, M. Bayer, A. Rahimi-Iman, A. Löffler, S. Höfling, S. Reitzenstein, L. Worschech, and A. Forchel, Proc. Natl. Acad. Sci. USA 108, 1804 (2011).

[14] V. Kohnle, Y. Léger, M. Wouters, M. Richard, M. T. PortellaOberli, and B. Deveaud-Plédran, Phys. Rev. Lett. 106, 255302 (2011).

[15] F. Li, L. Orosz, O. Kamoun, S. Bouchoule, C. Brimont, P. Disseix, T. Guillet, X. Lafosse, M. Leroux, J. Leymarie, M. Mexis, M. Mihailovic, G. Patriarche, F. Réveret, D. Solnyshkov, J. Zuniga-Perez, and G. Malpuech, Phys. Rev. Lett. 110, 196406 (2013).
[16] O. Jamadi, F. Réveret, E. Mallet, P. Disseix, F. Médard, M. Mihailovic, D. Solnyshkov, G. Malpuech, J. Leymarie, X. Lafosse, S. Bouchoule, F. Li, M. Leroux, F. Semond, and J. Zuniga-Perez, Phys. Rev. B 93, 115205 (2016).

[17] M. Nakayama, K. Murakami, Y. Furukawa, and D. Kim, Appl. Phys. Lett. 105, 021903 (2014).

[18] M. Nakayama, K. Murakami, and D. Kim, J. Phys. Soc. Jpn. 85, 054702 (2016).

[19] O. Madelung, Semiconductors: Data Handbook, 3rd ed. (Springer, Berlin, 2004), p. 103 for GaN, p. 194 for $\mathrm{ZnO,}$ p. 248 for $\mathrm{CuCl}$, and p. 254 for $\mathrm{CuBr}$.

[20] R. Butté, G. Delalleau, A. I. Tartakovskii, M. S. Skolnick, V. N. Astratov, J. J. Baumberg, G. Malpuech, A. Di Carlo, A. V. Kavokin, and J. S. Roberts, Phys. Rev. B 65, 205310 (2002).

[21] M. Nakayama, K. Miyazaki, T. Kawase, and D. Kim, Phys. Rev. B 83, 075318 (2011).

[22] M. Nakayama, Y. Kanatanai, T. Kawase, and D. Kim, Phys. Rev. B 85, 205320 (2012).

[23] M. H. Szymanska, J. Keeling, and P. B. Littlewood, Phys. Rev. Lett. 96, 230602 (2006).

[24] M. Wouters and I. Carusotto, Phys. Rev. Lett. 99, 140402 (2007).

[25] Y. Chen, A. Tredicucci, and F. Bassani, Phys. Rev. B 52, 1800 (1995).

[26] S. Suga, K. Cho, and M. Bettini, Phys. Rev. B 13, 943 (1976).

[27] M. S. Skolnick, T. A. Fisher, and D. M. Whittaker, Semicond. Sci. Technol. 13, 645 (1998).

[28] F. Médard, J. Zuniga-Perez, P. Disseix, M. Mihailovic, J. Leymarie, A. Vasson, F. Semond, E. Frayssinet, J. C. Moreno, M. Leroux, S. Faure, and T. Guillet, Phys. Rev. B 79, 125302 (2009).

[29] O. Svelto, Principles of Lasers, 5th ed. (Springer, New York, 2010), p. 163.

[30] Y. Unuma, Y. Masumoto, and S. Shionoya, J. Phys. Soc. Jpn. 51, 1200 (1982).

[31] T. Goto and M. Ueta, J. Phys. Soc. Jpn. 22, 488 (1967).

[32] G. Rossbach, J. Levrat, E. Feltin, J.-F. Carlin, R. Butté, and N. Grandjean, Phys. Rev. B 88, 165312 (2013).

[33] L. I. Schiff, Quantum Mechanics, 3rd ed. (McGraw-Hill, Singapore, 1968), p. 1. 